International Mathematical Forum, 1, 2006, no. 10, 455-469

\title{
Detecting Dependence in Heavy-Tailed Time Series using Portmanteau-type dependence tests
}

\author{
C. M. Gallagher \\ Dept. of Mathematical Sciences \\ Clemson University \\ Clemson SC, 29634-0975, U.S.A.
}

\begin{abstract}
We use the sample covariation to develop asymptotic tests for independence for data in the normal domain of attraction of a stable law. The tests can be used for finite or infinite variance processes. In a simulation study we compare the finite sample performance of the proposed tests to the Portmanteau test commonly used in time series modeling. The null convergence of the test statistics to their asymptotic distribution seems to be faster than that of the Portmanteau statistic, especially when the data is fat-tailed. In the finite variance case, the proposed tests have the same asymptotic power properties as the Portmanteau test and have similar small sample empirical power. Simulations indicate the covariation test has a higher power for small sample sizes when the process is symmetric stable with tail decay parameter $\alpha>1.6$.
\end{abstract}

Mathematics Subject Classification: 60G10, 60G52, 62M10.

Keywords: Stationary stochastic process, dependence, hypothesis test, infinite variance

\section{Introduction}

In this paper we generalize the dependence test of Gallagher (2002) to use higher order lags of the sample covariation (described below) to develop Portmanteau type tests for linear dependence in symmetric time series data. We compare the proposed tests to the traditional Portmanteau test for both infinite and finite variance data. We will see that the test statistics described below seem to converge to their null asymptotic distribution faster than the Portmanteau statistic, especially when the data is fat-tailed. In the finite variance case the proposed test shares similar power properties with the usual 
test. In the infinite variance case the proposed test can have significantly higher empirical power for small and moderate sample sizes in many cases.

Let $\left\{X_{t}\right\}$ be a strictly stationary ergodic stochastic process with zero mean satisfying

$$
\mathrm{E}\left(X_{t} \mid X_{t-k}\right)=\lambda(k) X_{t-k} \quad k=1,2, \ldots, h .
$$

Condition (1) is satisfied by any symmetric $\alpha$-stable process as well as any first order autoregression

$$
X_{t}=\phi X_{t-1}+Z_{t},
$$

where $\left\{Z_{t}\right\}$ is an independent identically distributed (iid) zero-mean sequence. For a zero-mean iid sequence $X_{t},(1)$ is satisfied with $\lambda(k)=0$ for $k=$ $1,2, \ldots, h$.

We consider testing the null hypothesis that $\left\{X_{t}\right\}$ is an iid sequence against

$$
\mathrm{H}_{1}: \quad \lambda(k) \neq 0 \quad \text { for some } \quad k=1,2, \ldots, h .
$$

Under $\mathrm{H}_{0}$ we will assume $X_{1}$ has a symmetric density in the normal domain of attraction (see Feller, 1971) of an $\alpha$-stable law for some $\alpha \in(1,2]$, i.e.,

$$
n^{(-1 / \alpha)} \sum_{t=1}^{n} X_{t} \Rightarrow S_{\alpha}
$$

where ' $\Rightarrow$ ' denotes convergence in distribution and $S_{\alpha}$ has a stable distribution with characteristic function

$$
E e^{\{i t S\}}=e^{-d|t|^{\alpha}}
$$

When $\alpha=2, \mathrm{E} X_{1}^{2}<\infty$ is a sufficient condition for (3).

If $X_{1}$ has a finite variance, the above hypothesis test is usually performed using the sample autocorrelation function $\hat{\rho}(j)$. For example, one may use the Portmanteau test statistic

$$
Q=n \sum_{j=1}^{h} \hat{\rho}^{2}(j)
$$

or the finite sample correction

$$
Q_{L B}=n(n+2) \sum_{j=1}^{h} \hat{\rho}^{2}(j) /(n-j),
$$

proposed by Ljung and Box (1978).

For $1<\alpha<2$, Runde (1997) considers using

$$
\left(\frac{n}{\ln n}\right)^{2 / \alpha} \sum_{j=1}^{h} \hat{\rho}^{2}(j),
$$


to test the iid hypothesis versus ARMA alternatives. This test assumes (3). Simulations indicate that the test performs poorly for small sample sizes, especially for $\alpha$ close to 2 . This is probably due to the apparent slow convergence of the normalized sample covariance and correlation functions to their asymptotic distributions when the underlying process has an infinite variance. This slow convergence rate was observed in Phillips and Loretan (1990) and Adler, Feldman and Gallagher (1999) as well.

We consider using the covariation function,

$$
\tau(k)=E X_{t} \delta_{t-k} \quad k=0, \pm 1, \pm 2, \ldots \pm h,
$$

where $\delta_{t}=\operatorname{sign}\left(X_{t}\right)$, and its sample version

$$
\hat{\tau}(k)=\left\{\begin{array}{ll}
n^{-1} \sum_{j=1}^{n-k} \delta_{t} X_{t+k} & \text { for } \quad k>0 \\
n^{-1} \sum_{j=1}^{n-k} X_{t} \delta_{t+k} & \text { for } \quad k \leq 0
\end{array},\right.
$$

to test the above hypothesis. For a process satisfying (1),

$$
\tau(k)=\lambda(k) E\left|X_{t-k}\right|,
$$

so that

$$
\lambda(k)=\frac{\tau(k)}{\tau(0)} .
$$

If the process is Gaussian, $\lambda(k)=\lambda(-k)=\rho(k)$.

Ergodicity implies that $\hat{\tau}(k)$ is a strongly consistent estimator of $\tau(k)$. Gallagher (2001) gives the asymptotic joint distribution of the sample covariation for ARMA processes with iid innovation sequences. If the innovation sequence satisfies (3) for some $\alpha \in(1,2]$,

$$
n^{(1-1 / \alpha)}(\hat{\tau}(k)-\tau(k)) \Rightarrow S_{\alpha},
$$

where $S_{\alpha}$ has a symmetric $\alpha$-stable distribution.

It is shown in Gallagher and Okuyama (2003) that for an iid sequence the univariate distribution function of the sample covariation converges at the same rate given in central limit theorems for iid processes. For example, if $\mathrm{E} X_{1}^{3}<\infty$, the Berry-Essen inequality applies. The rate of convergence for the sample covariation function seems to be faster than that corresponding to the covariance. In fact, if $\left\{X_{t}\right\}$ is an iid symmetric stable (Gaussian) process, $(n-k)^{(1-1 / \alpha)} \hat{\tau}(k)$ has an exact stable (Gaussian) distribution. We therefore expect hypothesis tests based on the sample covariation may perform well for small sample sizes.

In general $\tau(k) \neq \tau(-k)$, but if the process is iid with zero mean $\tau(k)=$ $\tau(-k)=0$. To test for independence we can define a symmetric covariation,

$$
T(k)=(\tau(k)+\tau(-k)) / 2
$$


which can be estimate with

$$
\hat{T}(k)=.5(\hat{\tau}(k)+\hat{\tau}(-k)) .
$$

In this paper we consider two test statistics. The first is based on the first $h$ positive lag covariations. When the process has an infinite variance we recommend the statistic

$$
Q_{\text {cov }}=\left(\hat{d}^{2}\right)^{-1} n^{(2-2 / \alpha)} \sum_{j=1}^{h} \hat{\tau}^{2}(j),
$$

where $\hat{d}$ is any consistent estimate of the scale $d$. If $\mathrm{E} X_{1}^{2}=\sigma^{2}<\infty$ we can improve the small sample power by using the first $h$ symmetric covariations,

$$
Q_{s}=\left(\hat{\mu}^{2}+\hat{\sigma}^{2}\right)^{-1} n \sum_{j=1}^{h} \hat{T}^{2}(j)
$$

where $\hat{\mu}$ is any consistent estimator for $\mathrm{E}\left|X_{1}\right|$. In the infinite variance case the null convergence of $Q_{s}$ (properly normalized) seems to be quite slow. Theorems 1.1 and 1.4 below give the asymptotic distributions of the statistics under the null hypothesis. Under the alternative hypothesis the test statistics diverge like $n^{(2-2 / \alpha)}$ as $n \rightarrow \infty$, so that the tests have classical $n^{(2-2 / \alpha)}$ consistency. The tests should have higher power as $\alpha \rightarrow 2$.

Theorem 1.1 Let $X_{1}, X_{2}, \ldots$ be a sequence of independent identically distributed symmetric random variables which belong to the normal domain of attraction of an $\alpha$-stable law with $\alpha \in(0,2]$. Let $h$ be an integer, then as $n \rightarrow \infty$,

$$
\left(\hat{d}^{2}\right)^{-1} n^{(2-2 / \alpha)} \sum_{j=1}^{h} \hat{\tau}^{2}(j) \Rightarrow k^{1-2 / \alpha} \mathbf{Y}^{\prime} \mathbf{A} \mathbf{Y}
$$

where $k=2^{h-1}, \mathbf{Y}$ is a vector of $h$ iid symmetric $\alpha$-stable random variables with scale $d=1$, and $\mathbf{A}$ is an idempotent matrix of rank $h$.

Remark 1.2 If $\mathrm{E} X_{1}^{2}=\sigma^{2}<\infty$ and $\hat{\sigma}^{2}$ is any consistent estimator, $n \hat{\sigma}^{-2} \sum_{j=1}^{h} \hat{\tau}^{2}(j)$ converges weakly to a chi-square distribution with $h$ degrees of freedom.

Remark 1.3 If $\left\{Y_{t}\right\}$ is not symmetric, the sequence $X_{2 t}=Y_{2 t}-Y_{2 t-1}$, can be used to test for independence. Under the null hypothesis, $\left\{X_{2 t}\right\}$ is iid with a symmetric density. If $Y_{t}$ is an $A R M A$ process, $Y_{t}=\sum_{j=0}^{\infty} \psi_{j} Z_{t-j}$ with $Z_{t}$ iid, then $X_{2 t}=Z_{2 t}-\sum_{j=1}^{\infty}\left(\psi_{j}-\psi_{j-1}\right) Z_{2 t-j}$. 
Theorem 1.4 Let $X_{1}, X_{2}, \ldots$ be a sequence of independent identically distributed symmetric random variables with $E X_{1}^{2}=\sigma^{2}<\infty$. Let $h$ be an integer, then as $n \rightarrow \infty$,

$$
\left(\hat{\mu}^{2}+\hat{\sigma}^{2}\right)^{-1} 2 n \sum_{j=1}^{h} \hat{T}^{2}(j) \Rightarrow \chi_{h}^{2},
$$

where $\chi_{h}^{2}$ denotes a chi-square random variable with $h$ degrees of freedom.

Remark 1.5 For $\alpha<2, n^{1-1 / \alpha} \sum_{j=1}^{h} \hat{T}^{2}(j)$ converges to a constant multiple of $\mathbf{Y}^{\prime} \mathbf{B} \mathbf{Y}$ where $\mathbf{B}$ is an idempotent matrix of rank $h$ and $\mathbf{Y}$ is a vector of $2^{2 h-1}$ iid symmetric stable variables, however initial simulations indicate the convergence under the null is quite slow.

For $\alpha<2$, Theorem 1.1 is impractical as it stands. In this case, the limiting distribution has no known closed form expression. In Section 2 we describe an algorithm for finding quantiles of the limiting distributions given above, and give quantiles for $h=3,5$, and 10 for various $\alpha$ 's.

In Section 3 we investigate the performance of the proposed tests on simulated data. We simulate power under $\operatorname{AR}(1)$ alternatives. In Section 3.1 we compare size and power of the test based on $Q_{\text {cov }}$ to the Runde (Portmanteau) statistic using simulated data from stable (normal) distributions. We first assume the scale parameter is known and simulate symmetric stable data for $\alpha<2$, and see that under the null hypothesis $Q_{\text {cov }}$ does not seem to be plagued with the slow distributional convergence of the sample covariance and correlation functions in the infinite variance case. In Section 3.2 we simulate from the normal and $t$-distribution (with 3 degrees of freedom) to compare empirical size and power for $Q_{\text {cov }}$ and $Q_{s}$ to that of the traditional statistic (4).

\section{Quantiles for $\alpha<2$}

In this section we consider estimating quantiles of the limiting distribution in Theorem 1.1 for $\alpha<2$. We give an algorithm for simulating random variates from the asymptotic distribution. To simulate quantiles, we used this algorithm to simulate 500,000 observations from the limiting distribution for each $\alpha=1.4,1.5,1.6,1.7,1.8,1.9$ and $h=3,5,10$.

The algorithm is easily implemented in S-plus. Table ?? gives the $90 \%$ and 95\% quantiles of $k^{1-2 / \alpha} \mathbf{Y}^{\prime} \mathbf{A Y}$ which were found via simulation using the $\mathbf{S}$ plus routine rstab to simulate symmetric stable variables. The code is available at http://www.ces.clemson.edu/ cgallag.

In order to give our algorithm we must first describe the matrix $\mathbf{A}$. Let $\delta=$ $\left(1, \delta_{2}, \ldots \delta_{h}\right)^{\prime}$. This vector takes $k=2^{h-1}$ equally likely possible values. Create 
Table 1: Quantiles of $k^{1-2 / \alpha} \mathbf{Y}^{\prime} \mathbf{A Y}$.

\begin{tabular}{lcccccc}
\hline & \multicolumn{2}{c}{$h=3$} & \multicolumn{2}{c}{$h=5$} & \multicolumn{2}{c}{$h=10$} \\
\hline$\alpha$ & .90 & .95 & .90 & .95 & .90 & .95 \\
\hline 1.4 & 31.57 & 78.03 & 52.33 & 129.98 & 103.35 & 259.91 \\
1.5 & 24.97 & 55.40 & 40.80 & 91.90 & 78.74 & 180.71 \\
1.6 & 20.43 & 40.99 & 32.78 & 65.97 & 63.27 & 129.72 \\
1.7 & 16.97 & 29.69 & 26.70 & 47.50 & 50.39 & 92.18 \\
1.8 & 14.82 & 22.64 & 22.42 & 34.59 & 40.73 & 64.38 \\
1.9 & 13.41 & 18.10 & 19.90 & 26.30 & 34.96 & 45.76 \\
2.0 & 12.50 & 15.63 & 18.47 & 22.14 & 15.99 & 18.31
\end{tabular}

A symmetric stable variable with $\alpha=2$ has variance 2 . The last row of the table is $2 * \chi_{h}^{2}(p)$ where $\chi_{h}^{2}(p)$ is the appropriate quantile of the chi-square distribution with $h$ degrees of freedom.

the matrix $\mathbf{M}$ by letting each column correspond to one of these outcomes. For example if $h=2$,

$$
\mathbf{M}=\left\{\begin{array}{rr}
1 & 1 \\
1 & -1
\end{array}\right\} .
$$

The joint limiting distribution of

$$
\hat{\tau}_{h}=(\hat{\tau}(1), \ldots, \hat{\tau}(h))^{\prime},
$$

is described in Proposition 2.1 below.

Proposition 2.1 Let $\left\{X_{t}\right\}$ be iid and satisfy (3). If $X_{1}$ has a symmetric density, the vector

$$
n^{1-1 / \alpha} \hat{\tau} \Rightarrow k^{-1 / \alpha} \mathbf{M Y},
$$

where $\mathbf{Y}$ is a vector of $k$ iid symmetric stable random variables each of which has scale $d$.

It is easy to see that $\mathbf{M}$ has orthogonal rows. It follows that

$$
\mathbf{A}=k^{-1} \mathbf{M}^{\prime} \mathbf{M},
$$

is an idempotent matrix. Using standard results from multivariate analysis, the quadratic form

$$
\mathbf{Y}^{\prime} \mathbf{A Y}=\mathbf{Y}^{\prime} \mathbf{P} \mathbf{P}^{\prime} \mathbf{Y},
$$

where the columns of $\mathbf{P}$ are the $h$ eigenvectors of $\mathbf{A}$ with non-zero eigenvalues. This suggests the following algorithm for simulating a sample of size $n$ from $\mathrm{Y}^{\prime} \mathrm{AY}$.

Algorithm 2.2 Create $\mathbf{M}$. This can easily be done recursively in $h$. Find the eigenvalues of $k^{-1} \mathbf{M}^{\prime} \mathbf{M}$ and create $\mathbf{P}$ For each $i=1,2, \ldots, n$ 
(i) Simulate $k$ symmetric stable random variables with index $\alpha$.

(ii) Set $y_{i}=k^{(1-2 / \alpha)} \mathbf{Y}^{\prime} \mathbf{P} \mathbf{P}^{\prime} \mathbf{Y}$.

\section{Empirical Results}

In this section we report the results of a simulation study. In the infinite variance case we simulated symmetric stable data. In the finite variance case we considered the $t$ distribution with 3 degrees of freedom and the normal distribution. Under the alternative hypothesis we simulated from autoregressive model (2) where $Z_{t}$ is an iid sequence with common symmetric $\alpha$-stable or $t$ density and $\phi=0.4$, and 0.7 .

In the infinite variance $(\alpha<2)$ case we will see that the proposed test statistics perform better as $\alpha$ approaches 2 . The problem with the tests for smaller $\alpha$ is that for small sample sizes the tests have low power. This is probably due to the slow divergence $\left(n^{2-2 / \alpha}\right)$ under the alternative hypothesis. For $\alpha \leq 1.5$ Runde's test is performing quite well - simulated type I error is essentially 0 and the test has good power properties. However, the covariation tests considered below seem to have better size and power properties than Runde's Portmanteau test for $\alpha>1.6$ and $n \leq 200$.

In the finite variance case the proposed test has the same asymptotic properties as the Portmanteau test. However, our simulations seem to indicate that the null convergence of $Q_{s}$ is faster than the convergence for $Q$ given by (4). The two tests seem to have comparable small sample power properties.

\subsection{Empirical size and power for symmetric stable data}

Here we compare the size and power of tests using $Q_{\operatorname{cov}}$ to that of the Portmanteau and Runde tests on simulated symmetric stable data. We will consider two different scale estimators $\hat{d}_{1}$ and $\hat{d}_{2}$ given below.

Table 2: Emperical size $\times 100$ for 0.05 tests when $d$ is known.

\begin{tabular}{lcccccc}
\hline & \multicolumn{2}{c}{$h=3$} & \multicolumn{2}{c}{$h=5$} & \multicolumn{2}{c}{$h=10$} \\
\hline$\alpha$ & $n=50$ & $n=100$ & $n=50$ & $n=100$ & $n=50$ & $n=100$ \\
1.5 & 4.92 & 4.49 & 4.89 & 4.77 & 4.56 & 4.74 \\
1.7 & 5.04 & 5.14 & 4.82 & 4.8 & 4.49 & 4.46 \\
1.9 & 4.53 & 4.84 & 4.82 & 4.79 & 4.29 & 5.24 \\
\hline
\end{tabular}

\subsubsection{Null convergence of $Q_{\operatorname{cov}}$ for symmetric stable data}

To investigate the null convergence of our test statistic in the infinite variance case, we simulated iid data from the symmetric stable distribution for various 
$\alpha$ values. Since the sample mean can converge quite slowly in this case, we used median-corrected data. Because distributional convergence can be quite slow for infinite variance sequences, we first investigate the null convergence of $\sum_{j=1}^{h} \tau(j)^{2}$ for $\alpha<2$ when the scale parameter is known. For symmetric stable data we consider two consistent estimators of $d$ and compare the proposed test to the Portmanteau test from Runde (1997). When $d$ is known, simulations indicate that the empirical size does not significantly depend on $\alpha$. In the case of unkown scale, for one of the scale estimators considered the distributional convergence seems to depend on $\alpha$ (slowing as $\alpha$ decreases).

For $d$ known, we report results for 10,000 tests for each $n=50,100$ and $\alpha=1.5,1.7$, and 1.9 in table 2 . We see that most of the numbers in the table are within 1.96 standard deviations of 0.05 . It appears that the test will be slightly conservative, since most of the empirical sizes are below 0.05 .

In the general case $d$ must be estimated using some robust (to moment assumptions) estimator. However, if the data is coming from a stable distribution, there are a number of estimators available. We consider two which should behave fairly well under the alternative hypothesis.

If $X_{1}$ has a symmetric stable distribution with scale $d$,

$$
E\left|X_{1}\right|=d 2 \Gamma(1-1 / \alpha) / \pi
$$

If the process generating the data sequence is stationary and ergodic with a finite mean the moment estimator

$$
\hat{d}_{1}=\frac{\pi \hat{\tau}(0)}{2 \Gamma(1-1 / \alpha)}
$$

is a strongly consistent estimator of $d$.

Under the null hypothesis,

$$
\left(\hat{\tau}(0)-E\left|X_{1}\right|\right)=O\left(n^{1 / \alpha-1}\right)
$$

which becomes increasingly slow as $\alpha$ approaches 1 . Thus we expect the test statistic using this moment estimator to perform better for $\alpha$ close to 2 . With this estimator of $d$, the test statistic becomes

$$
\hat{Q}_{\text {cov }}=n^{2-2 / \alpha} 2 \Gamma(1-1 / \alpha) / \pi \sum_{k=1}^{h} \hat{\lambda}^{2}(k),
$$

where

$$
\hat{\lambda}(k)=\frac{\hat{\tau}(k)}{\hat{\tau}(0)} .
$$

We note here that if (1) is satisfied,

$$
\hat{\lambda}(k) \rightarrow \lambda(k) .
$$


where the convergence is in the almost sure sense and $\lambda$ is given by (6).

We also consider the quantile estimator of McCulloch (1986). This estimator was originally proposed for iid data and is a consistent estimator in this case. Since the estimator uses the sample quantiles, we expect it to work under the alternative hypothesis as well. Indeed Adler, Feldman, and Gallagher (1998) give simulation evidence indicating that the quantile estimator of the tail decay parameter $\alpha$ works well for small order moving average and autoregressive processes. For this scale estimator we denote the test statistic by

$$
\tilde{Q}_{\text {cov }}=n^{2-2 / \alpha} \sum_{k=1}^{h} \frac{\hat{\tau}^{2}(k)}{\hat{d}_{2}^{2}},
$$

where $\hat{d}_{2}$ denotes McCulloch's quantile estimator of $d$.

Table 3 contains simulated size for the tests based on $\hat{Q}_{c o v}, \tilde{Q}_{c o v}$, and $Q$. For each $n$ and $\alpha$ we simulated 1,000 data sets under the null hypothesis with $d=3$.

Table 3: Emperical size $\times 100$ for 0.05 tests when $d$ is unknown.

\begin{tabular}{lccccccccc}
\hline & \multicolumn{3}{c}{$n=50$} & \multicolumn{3}{c}{$n=100$} \\
$\alpha$ & $\hat{Q}_{\text {cov }}$ & $\tilde{Q}_{\text {cov }}$ & $Q$ & $\hat{Q}_{\text {cov }}$ & $\tilde{Q}_{\text {cov }}$ & $Q$ & $\hat{Q}_{\text {cov }}$ & $\tilde{Q}_{\text {cov }}$ & $Q$ \\
1.5 & 1.6 & 5.5 & 0 & 1.4 & 4.7 & 0 & 2.4 & 5.3 & 0.1 \\
1.6 & 1.2 & 6.5 & 0 & 2.1 & 6 & 0 & 2.6 & 5.5 & 0 \\
1.7 & 1.4 & 5.2 & 0 & 2.7 & 4.9 & 0 & 3.1 & 5.5 & 0 \\
1.8 & 2.2 & 5.3 & 0 & 3.5 & 6.3 & 0 & 3.8 & 5.3 & 0 \\
1.9 & 3.3 & 5.4 & 0 & 3.7 & 5.1 & 0 & 3.6 & 5.3 & 0 \\
2.0 & 3.9 & 6.8 & 5.1 & 5.1 & 6.6 & 4.6 & 5.5 & 5.7 & 6 \\
\hline
\end{tabular}

For $\alpha<2$, the statistic $\tilde{Q}_{\text {cov }}$ seems to perform best, since all but 1 of its empirical sizes are within $.05 \pm 1.96 \sqrt{.95 * .05 / 1000}$. The test based on $\hat{Q}_{\text {cov }}$ and Runde's Portmanteau test appear to be conservative in the sense that the empirical size is systematically below its nominal value. The Portmanteau test essentially had 0 type I error for the infinite variance data, while $\hat{Q}_{\text {cov }}$ has simulated sizes which seem to be approaching 0.05 as $\alpha$ and $n$ increase.

\subsubsection{Empirical power for symmetric stable data.}

We conducted a small simulation study to assess the finite sample power properties of the proposed test. We considered both scale estimators mentioned above and compared the performance to that of the test proposed in Runde (1997). For comparison purposes we assumed $\alpha$ was known (We only had quantiles for Runde's test for specific $\alpha$ values). In practice $\alpha$ can be estimated using McCulloch's quantile method or any of the numerous methods available in the literature. 
For each $\alpha$ and $n=50,100,200$ we simulated 1,000 data sets from each of the two autoregressive models mentioned above. We compare empirical power for $\hat{Q}_{c o v}, \tilde{Q}_{c o v}$, and $Q$ given by (12), (13), and (5) respectively. Quantiles for the limiting distribution of (5) are given in Runde (1997) for various $\alpha \in(1,2)$ and $h=2,3,4$ and 5 .

Table 4: Percent rejected (power) of 0.05-level tests when $d$ is unknown.

\begin{tabular}{lcccccccccc}
\hline & \multicolumn{4}{c}{$n=50$} & \multicolumn{3}{c}{$n=100$} & \multicolumn{3}{c}{$n=200$} \\
Model & $\alpha$ & $\hat{Q}_{\text {cov }}$ & $\tilde{Q}_{\text {cov }}$ & $Q$ & $\hat{Q}_{\text {cov }}$ & $\tilde{Q}_{\text {cov }}$ & $Q$ & $\hat{Q}_{\text {cov }}$ & $\tilde{Q}_{c o v}$ & $Q$ \\
\hline$\phi=0.7$ & 1.5 & 9.9 & 12.1 & 59.8 & 36.2 & 22.3 & 98.6 & 79.7 & 53.4 & 99.9 \\
$\phi=0.4$ & & 1.4 & 6.4 & 1.0 & 1.6 & 6.6 & 13.5 & 6.8 & 9.7 & 85.1 \\
$\phi=0.7$ & 1.6 & 25.7 & 19.2 & 33.8 & 65.6 & 48.9 & 95.1 & 97.6 & 93.6 & 99.8 \\
$\phi=0.4$ & & 2.8 & 6.5 & 0.1 & 5.4 & 8.5 & 3.7 & 15.7 & 15.4 & 45.9 \\
$\phi=0.7$ & 1.7 & 53.5 & 41.4 & 21.4 & 89.1 & 84.7 & 82.8 & 99.9 & 99.9 & 99.9 \\
$\phi=0.4$ & & 8.2 & 11.1 & 0 & 19.3 & 19.8 & 0.5 & 48.3 & 44.1 & 17.3 \\
$\phi=0.7$ & 1.8 & 71.1 & 64.5 & 5.7 & 98.1 & 97.3 & 63.2 & 1 & 1 & 99.5 \\
$\phi=0.4$ & & 14.2 & 15.5 & 0 & 38.7 & 36.9 & 0.1 & 77.9 & 73.4 & 2.9 \\
$\phi=0.7$ & 1.9 & 86.0 & 82.1 & 01.6 & 99.6 & 99.1 & 38.9 & 1 & 1 & 97.3 \\
$\phi=0.4$ & & 30.4 & 29.0 & 0 & 59.1 & 58.2 & 0 & 93.3 & 92.5 & 0.5 \\
$\phi=0.7$ & 2.0 & 91.9 & 90.5 & 98.0 & 99.9 & 99.9 & 1 & 1 & 1 & 1 \\
$\phi=0.4$ & & 38.3 & 38.8 & 55.0 & 72.6 & 72.9 & 89.4 & 98.0 & 984 & 99.9 \\
\hline
\end{tabular}

For $n=50$ we took $h=3$ and for $n=100,200$ we used $h=5 . \alpha=2$ corresponds to the normal distribution and $Q$ given by (4).

As mentioned above the proposed tests give better results for $\alpha$ closer to 2 . Our simulations indicate that the covariation tests seem to have a higher power than Runde's test for small to moderate $n$ and $\alpha>1.6$, with a significantly higher power for $\alpha \in[1.7,2)$. For $\alpha \leq 1.6$ Runde's test seems to have a higher power. This phenomenon can be seen by results given in Table 4 . It is not clear which scale estimator is performing better. It is not too surprising that for normal data $(\alpha=2)$, the Portmanteau test has higher empirical power for all $n$ considered.

\subsection{Simulations for finite variance data}

To assess the small sample size and power properties of the tests for some finite variance distributions, we performed a small simulation study. We compared the Portmanteau test statistic (4) to $Q_{\text {cov }}$ given by (8), as well as to $Q_{s}$ given by (9). For $Q_{s}$ we used simple moment estimators of $\mu=\mathrm{E}\left|X_{1}\right|$ and $\sigma^{2}$, while we used the sample variance in place of $\hat{d}$ in (8). Since the test statistic $Q_{s}$ had better empirical size and power than $Q_{c o v}$, for brevity we report results only for this statistic. 
To compare empirical sizes for the Portmanteau and covariation tests, we simulated under the null from the normal distribution with variance 9 as well as from the $t$ distribution with 3 degrees of freedom. For each $n=10,20,50$ and 100, we simulated 10000 data sets from each distribution and counted the number of rejections from each test statistic for $h=5$. The results appear below in Table 5 . We see that the simulated sizes for $Q_{s}$ are closer to 0.05 for all $n$ considered for both normal as well as $t$ data.

Table 5: Emperical size $\times 100$ of 0.05 tests for normal and $t$ densities.

\begin{tabular}{lcccccccc}
\hline & \multicolumn{2}{c}{$n=10$} & \multicolumn{2}{c}{$n=20$} & \multicolumn{2}{c}{$n=50$} & \multicolumn{2}{c}{$n=100$} \\
density & $Q$ & $Q_{s}$ & $Q$ & $Q_{s}$ & $Q$ & $Q_{s}$ & $Q$ & $Q_{s}$ \\
\hline normal & 0.48 & 1.08 & 2.18 & 2.85 & 3.30 & 3.99 & 4.57 & 4.70 \\
$\mathrm{t}$ & 0.29 & 1.03 & 1.30 & 2.97 & 2.73 & 4.08 & 3.63 & 4.49 \\
\hline
\end{tabular}

Table 6 contains empirical power for 1000 data sets simulated under the two autoregressive processes considered above. We see that not only do the two tests have the same asymptotic power properties, the small sample empirical power is quite similar for both statistics. The proposed test may work slightly better for smaller sample sizes and the Portmanteau test appears to give slightly higher power for moderate sample sizes.

Table 6: Percent rejected (power) for 0.05 tests.

\begin{tabular}{|c|c|c|c|c|c|c|c|c|c|}
\hline \multirow[b]{2}{*}{ density } & \multirow[b]{2}{*}{ Model } & \multicolumn{2}{|c|}{$n=10$} & \multicolumn{2}{|c|}{$n=20$} & \multicolumn{2}{|c|}{$n=50$} & \multicolumn{2}{|c|}{$n=100$} \\
\hline & & $Q$ & $Q_{s}$ & $Q$ & $Q_{s}$ & $Q$ & $Q_{s}$ & & \\
\hline $\bar{t}$ & $\phi=0.7$ & 0 & 1.0 & 40.1 & 40.8 & 96.6 & 95.2 & 1.0 & 1.0 \\
\hline & $\phi=0.4$ & 0 & 0.2 & 7.3 & 11.6 & 45.7 & 45.4 & 86.7 & 82 \\
\hline normal & $\phi=0.7$ & 0.2 & 1.1 & 38.5 & 38.3 & 96.5 & 92.5 & 1.0 & 1.0 \\
\hline & $\phi=0.4$ & 0.2 & 0.6 & 9.0 & 9.6 & 45.3 & 39.7 & 86.7 & 77.4 \\
\hline
\end{tabular}

For all $n$ we used $h=5$.

\section{Proof of the Main Results.}

We derive the weak limit of $Q_{\text {cov }}$ and $Q_{s}$ under the null hypothesis. The limiting distributions under ARMA alternatives follow from Theorem 2.2 in Gallagher (2001) via the continuous mapping theorem. We will deal with the finite and infinite variance cases seperately.

\subsection{Finite variance case.}

For $\alpha=2$, Theorem 1.1 is an immediate consequence of Proposition 4.1. 
Proposition 4.1 Let $\left\{X_{t}\right\}$ be an iid symmetric mean zero process with $E X_{1}^{2}<$ $\infty$. For integer $h$,

$$
\sqrt{n} \hat{\tau}_{h} \Rightarrow \mathbf{Z}
$$

where $\mathbf{Z}$ is a vector of iid normal random variables each of which has variance $E X_{1}^{2}$.

proof Let $\left(a_{1}, \ldots, a_{h}\right)^{\prime} \in \Re^{h}$ and define

$$
Y_{t}=X_{t}\left(a_{h} \delta_{t-h}+\cdots+a_{1} \delta_{t-1}\right) .
$$

Stationarity of $Y_{t}$ follows from the fact that the process $X_{t}$ is stationary. Using the symmetry and independence assumptions we see $Y_{t}$ is an h-dependent process with covariance function

$$
\gamma(k)= \begin{cases}E X_{1}^{2} \sum_{j=1}^{h} a_{j}^{2} & k=0 \\ 0 & k=1, \ldots, h\end{cases}
$$

Using the central limit theorem for stationary m-dependent processes (e.g. see Brockwell and Davis, 1991), we see that

$$
n^{-1 / 2} \sum_{t=1}^{n} Y_{t} \Rightarrow N
$$

where $N$ has a normal distribution with variance $E X_{1}^{2} \sum_{j=1}^{h} a_{j}^{2}$.

Theorem 1.4 follows directly from Proposition 4.2.

Proposition 4.2 Let $\left\{X_{t}\right\}$ be an iid symmetric mean zero process with $E X_{1}^{2}=$ $\sigma^{2}<\infty$ and $c^{2}=\left(E\left|X_{1}\right|\right)^{2}$. For integer $h$ let

$$
\mathbf{T}^{t}=(T(1), \ldots, T(h)),
$$

The vector

$$
\sqrt{n} \mathbf{T} \Rightarrow \mathbf{Z}
$$

where $\mathbf{Z}$ is a vector of iid normal random variables each of which has variance $\left(\sigma^{2}+c^{2}\right) / 2$.

proof Let $\left(a_{1}, \ldots, a_{h}\right)^{\prime} \in \Re^{h}$ and define

$$
Y_{t}=X_{t} / 2\left(a_{h}\left(S_{t-h}+S_{t+h}\right)+\cdots+a_{1}\left(S_{t-1}+S_{t+1}\right)\right) .
$$

Using the symmetry and independence assumptions we see $Y_{t}$ is an h-dependent process with covariance function

$$
\gamma(k)= \begin{cases}\sigma^{2} / 2 \sum_{j=1}^{h} a_{j}^{2} & k=0 \\ \left(a_{k} E\left|X_{1}\right|\right)^{2} / 4 & k=1, \ldots, h\end{cases}
$$


Using the central limit theorem for stationary m-dependent processes (e.g. see Brockwell and Davis, 1991), we see that

$$
n^{-1 / 2} \sum_{t=1}^{n} Y_{t} \Rightarrow N
$$

where $N$ has a normal distribution with variance

$$
v_{h}=\frac{\sigma^{2}+\left(E\left|X_{1}\right|\right)^{2}}{2} \sum_{j=1}^{h} a_{j}^{2} .
$$

\section{2 infinite variance data}

For $\alpha<2$ Theorem 1.1 is an immediate consequence of Proposition 2.1.

proof of Proposition 2.1 For $\mathbf{a}=\left(a_{1}, \ldots, a_{h}\right)^{\prime} \in \Re^{h}$, define

$$
Y_{t}=d^{-1} X_{t}\left(a_{h} \delta_{t-h}+\cdots+a_{1} \delta_{t-1}\right)
$$

$Y_{t}$ is an h-dependent symmetric process with

$$
n P\left(\left|Y_{1}\right|>n^{1 / \alpha} x\right) \rightarrow c_{\mathbf{a}} x^{-\alpha} \quad \text { as } \quad x \rightarrow \infty
$$

where $c_{\mathbf{a}}$ is a constant given below. To describe $c$ let the vector

$$
\delta_{h}=\left(\delta_{1}, \ldots, \delta_{h}\right)^{\prime}
$$

Then

$$
c_{\mathbf{a}}=E\left|\mathbf{a}^{\prime} \delta_{h}\right|^{\alpha} .
$$

The sequence $\left\{Y_{t}\right\}$ satisfies all three conditions of Davis (1983). Condition D is implied by mixing and condition $\mathbf{D}^{\prime \prime}$ follows from $h$-dependence. To see that $\mathbf{D}^{\prime}$ holds note that

$$
n P\left(Y_{1}>n^{1 / \alpha} x, Y_{j}>n^{1 / \alpha} x\right) \leq n\left(P\left(\left|X_{1}\right|>C_{1} n^{1 / \alpha} x\right)\right)^{2}
$$

where $C_{1}=\sum\left|a_{j}\right|$. Applying Theorems 2 and 3 in Davis (1983), we see that the partial sums

$$
n^{-1 / \alpha} \sum_{t=1}^{n} Y_{t} \Rightarrow W
$$

where $W$ has a symmetric stable distribution with scale $c_{\mathbf{a}}$. The vector $\delta$ has $2^{h}$ unique possible outcomes. Meaning that $\left|\mathbf{a}^{\prime} \delta\right|$ has at most $k=2^{h-1}$ unique (equally likely) outcomes. Each of these outcomes corresponds to a 
column of M. Using well known properties of stable random variables (e.g. see Sammorodnitsky and Taqqu, 1994) it follows that

$$
n^{-1 / \alpha} \sum_{t=1}^{n} Y_{t} \Rightarrow k^{-1 / \alpha} \mathbf{a}^{\prime} \mathbf{M Y}
$$

where $\mathbf{Y}$ is a vector consisting of $k$ iid symmetric stable random variables with scale 1.

Remark 4.3 The limiting multivariate stable vector MY in Proposition 2.1 has spectral measure concentrated on $k$ symmetric pairs of points on the unit circle in $\Re^{h}$. Unlike the finite variance case this limiting vector does not have independent components.

\section{References}

[1] R. J.Adler, R. Feldman, and C. Gallagher, Analysing stable time series, in "A Practical Guide to Heavy Tails" (R. J. Adler, R. E. Feldman, and M. S. Taqqu, eds.), Birkhäuser, Boston, 1998.

[2] P. J. Brockwell and R. A. Davis, Time Series: Theory and Methods, Springer, New York, 1991.

[3] R. A. Davis, Stable limits for partial sums of dependent random variables, Ann. Probab., Vol. 11 (1983), 262-269.

[4] W. Feller, An Introduction to Probability Theory and its Applications, Wiley, New York, 1971.

[5] C. Gallagher, Estimating the autocovariation from stationary heavytailed data, with applications to time series modeling (2001), Dept. of Mathematical Sciences technical report Gal0101 available at www.ces.clemson.edu/ cgallag/tech.html.

[6] C. Gallagher and T. Okuyama, Some differences in the rates of convergence of the sample covariance and covariation functions. Interstat (2003)

[7] C. Gallagher, Testing for Linear Dependence in Heavy-Tailed Data. Comm. in Stat.: Theory and Methods, 31, 4, (2002).

[8] G. M. Ljung, and G. E. P. Box, On a measure of lack of fit in time series models, Biometrika, Vol. 65 (1978), 297-303.

[9] J.H. McCulloch, Simple consistent estimators of stable distribution parameters, Communications in Statistics - Computation and Simulation, Vol. 15 (1986), 1109-1136. 
[10] P. C. B. Phillips, and M. Loretan, The Durbin-Watson ratio under infinitevariance errors, J. of Econometrics, Vol. 47 (1991), 85-114.

[11] R. Runde, The asymptotic null distribution of the Box-Pierce Q-statistic for random variables with infinite variance, J. of Econometrics 78 (1997), $205-216$.

[12] G. Samorodnitsky, and M. Taqqu, Stable Non-Gaussian Random Processes, Chapman-Hall, New York, 1994.

Received: August 21, 2005 